Results Figure 1.185 patients were reviewed and 23 excluded. $85 \%$ of patients had their smoking status clearly documented. Only $30 \%$ of smokers were provided with cessation advice and 7\% were referred to Quitters. 69.5\% of smokers did not have NRT prescribed but only $19.5 \%$ not wish to receive NRT.

Conclusions The medical impatient population has a higher prevalence of smokers (28\%) when compared to the national average of $21 \%$. Our results for smoking cessation service provision compare poorly with NICE guidelines which target $100 \%$ of smokers receiving smoking cessation guidance.

Awareness needs to be raised amongst the medical staff in order to optimise the provision of advice, referral to quitters and NRT prescription rate in order to achieve the targets set by NICE and the $\mathrm{DOH}$. We are currently embarking upon a series of educational sessions and a ward round 'checklist' is under development which will include a prompt for smoking cessation.

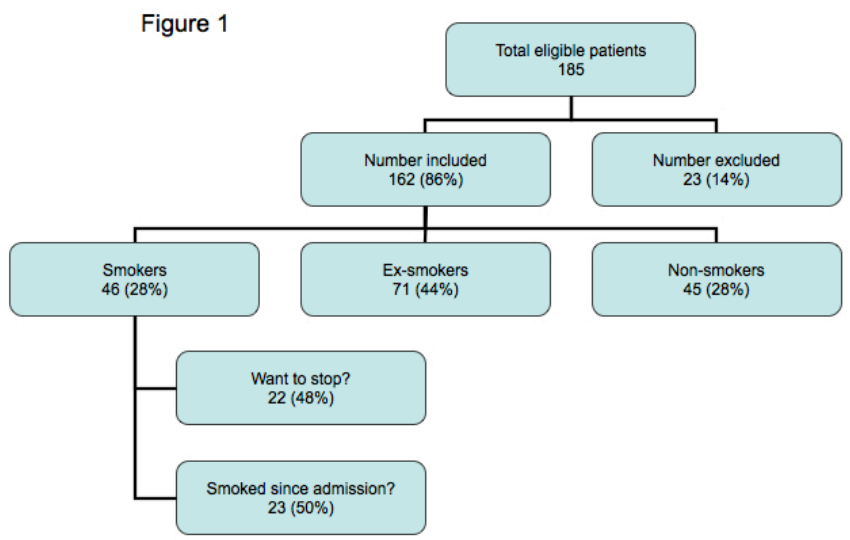

Abstract P205 Figure 1

\section{Clinical management of patients with COPD}

\section{P206 THE ACCEPTABILITY OF STARTING NON-INVASIVE VENTILATION AT HOME TO PATIENTS WITH COPD AND THEIR CARERS}

doi:10.1136/thoraxjnl-2012-202678.267

M Jawad, NK Gale, AM Turner. University of Birmingham, Birmingham, United Kingdom

Introduction Home non-invasive ventilation (NIV) is prescribed to patients suffering from severe chronic obstructive pulmonary disease (COPD) with chronic respiratory failure. Uncertainty exists regarding clinical efficacy and the effect on a patient's quality of life (OOL) during long term use. This qualitative study aimed to explore the perceptions and experiences of healthcare professionals (HCPs) involved in the management of COPD patients, COPD patients themselves and the patient's carers regarding the use of home NIV, with the aim of understanding decision making processes and improving its future use.

Method 15 HCPs including doctors, nurses, physiotherapists and physiologists, 20 COPD patients with moderate to very severe disease and 4 carers ( 3 patient spouses and 1 patient sibling) were recruited to participate in a semi-structured interview from a tertiary NIV hospital, a hospital providing home NIV services and the community. All interviews were transcribed verbatim and analysed using framework analysis.

Results Two major themes emerged from the interviews. The need for 'adapting to home NIV' was reported by all patients. This corresponds to the need for acceptance of the NIV and the ability to overcome NIV specific problems. Patients expressed that this change was forced upon them by their HCPs. It was evident from the interviews that carers also experienced this need for adaptation. Another theme that emerged from the interviews was of 'negotiating the evidence'. HCPs reported that due to the lack of evidence and guidance available for home NIV use, they encountered difficulties when deciding which patients to start or continue on home NIV. Patients faced a dilemma of whether to accept the doctor's 'orders' to use home NIV, despite distressing NIV experiences at hospital. Nevertheless improvements in the patient's QOL, particularly in their day-to-day functions, were reported.

Conclusion This study demonstrated that patients find the use of home NIV acceptable once they have adapted to it. Recommendations include providing patients with greater technical NIV support, a better experience of NIV at hospital and improving communication between HCPs and patients to allow for greater understanding of the patient's perspective.

\section{P207 CAN HOLISTIC INTERVENTIONS IMPROVE THE CARE OF PEOPLE WITH SEVERE CHRONIC OBSTRUCTIVE PULIMONARY DISEASE (COPD)? A SYSTEMATIC REVIEW}

doi:10.1136/thoraxjnl-2012-202678.268

UB Nurmatov, S Buckingham, M Kendall, A Sheikh, H Pinnock. The University of Edinburgh, Edinburgh, Scotland, UK

Introduction and Objectives People with severe COPD have a long-recognised burden of disabling physical symptoms compounded by co-morbidity, psychological distress and social isolation. We aimed to review the effectiveness of interventions designed to deliver holistic care compared to usual care for people with severe COPD.

Methods We searched 11 international electronic databases, three trial repositories and contacted a panel of international experts to locate published, unpublished and in-progress randomised controlled trials (RCTs), quasi-RCTs and controlled clinical trials (CCTs) that investigated holistic interventions designed to support patients with severe COPD in any healthcare context. Date range January 1990-March 2012; no language or geographical restrictions. Quality assessment and data extraction followed the Cochrane Collaboration method. Health-related quality of life (HROoL) was the primary outcome. We used a piloted data extraction sheet and undertook narrative synthesis.

Results From 2,866 potentially relevant papers, three trials met our inclusion criteria: two RCTs (from United States and Australia), and one CCT (from Thailand) studying a total of 216 patients. Critical appraisal identified a moderate (one RCT and the CCT) or high (one RCT) risk of bias. All interventions were led by nurses acting in a co-ordinating role (e.g. facilitating community support in Thailand, providing case-management in the US, or co-ordinating inpatient care in Australia). The community-based intervention in Thailand significantly improved HRQoL at three months compared to (limited) usual care (St George's Respiratory Questionnaire: intervention: $30.3 \pm 19.4$ vs control $52.4 \pm 21.3 p<0.001)$. Significant effects in the US trial were confined to 'Physical functioning' and 'General health' sub-domains of Medical Outcomes Study ShortForm-36 at three but not six-months. There were no significant changes in the Australian trial.

Conclusions Some 15 years after reports first highlighted the substantial unmet needs of people with severe COPD, we have been unable to find robust trial evidence about interventions to address those needs. There is an urgent need to develop and evaluate interventions to deliver or enhance holistic care and improve the quality of life of people with severe COPD

Funding Dunhill Medical Trust. HP is supported by a PCRCA Fellowship (CSO, Scottish Government). 